ARTICLE

\title{
Cascaded emission of single photons from the biexciton in monolayered $\mathrm{WSe}_{2}$
}

\author{
Yu-Ming He ${ }^{1,2}$, Oliver Iff', Nils Lundt ${ }^{1}$, Vasilij Baumann', Marcelo Davanco ${ }^{3}$, Kartik Srinivasan ${ }^{3}$, Sven Höfling ${ }^{1,4}$ \\ $\&$ Christian Schneider ${ }^{1}$
}

Monolayers of transition metal dichalcogenide materials emerged as a new material class to study excitonic effects in solid state, as they benefit from enormous Coulomb correlations between electrons and holes. Especially in $\mathrm{WSe}_{2}$, sharp emission features have been observed at cryogenic temperatures, which act as single photon sources. Tight exciton localization has been assumed to induce an anharmonic excitation spectrum; however, the evidence of the hypothesis, namely the demonstration of a localized biexciton, is elusive. Here we unambiguously demonstrate the existence of a localized biexciton in a monolayer of $\mathrm{WSe}_{2}$, which triggers an emission cascade of single photons. The biexciton is identified by its time-resolved photoluminescence, superlinearity and distinct polarization in micro-photoluminescence experiments. We evidence the cascaded nature of the emission process in a cross-correlation experiment, which yields a strong bunching behaviour. Our work paves the way to a new generation of quantum optics experiments with two-dimensional semiconductors.

\footnotetext{
${ }^{1}$ Technische Physik and Wilhelm-Conrad-Röntgen Research Center for Complex Material Systems, Universität Würzburg, Würzburg, Am Hubland, D-97074 Germany. ${ }^{2}$ Hefei National Laboratory for Physical Sciences at the Microscale, Department of Modern Physics and CAS Center for Excellence and Synergetic Innovation Center in Quantum Information and Quantum Physics, University of Science and Technology of China, Hefei, Anhui 230026, China. ${ }^{3}$ Center for Nanoscale Science and Technology, National Institute of Standards and Technology, Gaithersburg, Maryland 20899, USA. ${ }^{4}$ SUPA, School of Physics and Astronomy, University of St Andrews, St Andrews KY16 9SS, UK. Correspondence and requests for materials should be addressed to C.S. (email: christian.schneider@physik.uni-wuerzburg.de).
} 
ncreasing interest and need for secure communications ${ }^{1}$, precision measurements, metrology ${ }^{2}$ and optical quantum emulation $^{3}$ explain the ever growing demand for non-classical sources of light. Ultra-compact implementations of such sources in solid state are particularly interesting, as they promise longterm stability, and outline possible ways for scalable integration, even in complex on chip quantum networks ${ }^{4}$. Regarding compactness, the ultimate limit is represented by a zerodimensional emission centre of non-classical light in atomic monolayers $^{5}$. Transition metal dichalcogenides semiconductors have emerged as a new platform to study excitonic effects in two dimensions ${ }^{6-10}$, in particular as they benefit from enormous Coulomb correlations between electrons and holes as a result of reduced dielectric screening ${ }^{11-13}$ and feature unique spinor properties ${ }^{14}$. Strong progress has been made in studying excitonic effects in two-dimensional materials, including the exploration of the valley pseudospin dynamics ${ }^{14,15}$, valley polarization control $^{16-20}$ and strong coupling effects ${ }^{21,22}$. Although single photon emission from localized states in monolayers of transition metal dichalcogenides has recently been observed ${ }^{23-27}$, the characteristic feature that determines an anharmonic excitation spectrum in an excitonic system, which is a well-isolated biexcitonic state, has not been observed. In addition, strong spectral wandering and even blinking, induced by a supposedly noisy environment, made it debatable whether the full potential of such ultra-compact solid-state single photon sources or pair sources based on mono-atomic layers can be harnessed. Recently, delocalized biexcitonic states in $\mathrm{WSe}_{2}$ have been attributed to a superlinearly increasing, broad emission feature on the lowenergy side of the characteristic excitonic resonance ${ }^{28}$. However, these indications clearly outline the need for further unambiguous evidence of multiexcitonic complexes in monolayer materials.

Here we demonstrate the existence of a localized biexciton in a monolayer of $\mathrm{WSe}_{2}$ at cryogenic temperatures. The optical properties of the localized excitons are strongly improved by transferring them onto epitaxially grown semiconducting material. We routinely observe sharp emission features with linewidth $<70 \mu \mathrm{eV}$ by means of non-resonant photoluminescence spectroscopy. The localized exciton-biexciton pair is identified by its characteristic polarization, the characteristic power dependency and, most importantly, the emission cascade is observed in single-photon correlation studies.

\section{Results}

Device description and optical characterization. Figure 1a depicts a graphic illustration of our investigated device: it consists of a $250 \mathrm{~nm}$-thick GaInP layer, which has been grown on a semi-insulating GaAs substrate via Gas-Source molecular beam epitaxy. A single layer of $\mathrm{WSe}_{2}$, mechanically exfoliated via commercial adhesive tape (Tesa brand) from a bulk crystal was transferred onto the atomically smooth and chemically inert GaInP layer with a polymer stamp. We did not apply any capping technique to the monolayer, to take advantage of the light extraction from the surface allocated emitters. Figure $1 \mathrm{~b}$ illustrates a two-dimensional scanning image of the photoluminescence emission in the energy range between 1.525 and $1.734 \mathrm{eV}$. The bulk shape could be reproduced by the suppressed photoluminescence from the substrate in the energy range $1.525-1.55 \mathrm{eV}$ and the localized shining spots show the sharp peaks emission, which are randomly found at the edge of the flake.

A series of non-resonant micro-photoluminescence spectra recorded at varying pump powers is shown in Fig. 1c). The single quantum emitter, which was excited by a $532 \mathrm{~nm}$ continuous-wave (CW) laser at a sample temperature of nominally $4.5 \mathrm{~K}$, exhibits two pronounced emission features with a distinctly different power dependency. We note that the two emission features, which have an energetic separation of $4.6 \mathrm{meV}$, have a spectral linewidth that is limited by the resolution of our spectrometer $(70 \mu \mathrm{eV})$ at lowest pump powers. This outlines the excellent optical properties of our localized emitters, which are not affected by obvious effects of long-term spectral wandering, as commonly observed for $\mathrm{WSe}_{2}$ emitters on insulating substrates ${ }^{23-27}$. More details concerning the optical quality of our quantum emitters can be found in Supplementary Fig. 1 and Supplementary Note 1. As we increase the pump power, the linewidth of our emission features steadily increases, which we attribute to power-induced dephasing ${ }^{29}$ (see Fig. 1d). More importantly, as shown in Fig. 1e, we observe a distinctively different behaviour of the integrated intensity of the two features, as we increase the excitation power. The intensity of the high-energy signal (P2) increases less rapidly than the low-energy signal (P1), and a characteristic saturation behaviour of $\mathrm{P} 1$ is present as $\mathrm{P} 2$ still increases in intensity. We fit our data with a power law $I \sim P^{x}$ and find a power law coefficient of $x=0.84$ for $\mathrm{P} 2$ and a superlinear coefficient of $x=1.42$ for $\mathrm{P} 1$. This pronounced sub- and superlinear behaviour of the two emission features already outlines a possible emission cascade, which is initialized by a biexcitonic state. With increasing sample temperature, $\mathrm{P} 1$ and $\mathrm{P} 2$ experience a characteristic broadening of the emission lines and a quenching of their intensity, which is detailed in the Supplementary Fig. 2 and Supplementary Note 2. A second pair of emission lines, which we found in our monolayer and which behaves qualitatively similar, is depicted in Supplementary Fig. 3.

The exciton and biexciton could be further identified by the time-resolved photoluminescence of the localized emitter excited with a 3 ps pulsed laser at $475 \mathrm{~nm}$ (see Fig. 1f and Supplementary Fig. 4). Both P1 and P2 were fitted with a single exponential decay function with the time constant of $\tau=0.793 \pm 0.017 \mathrm{~ns}$ for $\mathrm{P} 1$ and $\tau=1.504 \pm 0.028 \mathrm{~ns}$ for P2. For the exciton and biexciton in semiconductor quantum dots, the time evolution of the probabilities for exciton (X) and biexciton (XX) could be modelled with the radiative transition functions ${ }^{30}$ and the ratio $\tau_{\mathrm{x}} / \tau_{\mathrm{xx}}$ would be dependent on their electron-hole spatial wave function separation. A ratio $\tau_{\mathrm{x}} / \tau_{\mathrm{xx}}=2$ is expected for excitons in the presence of a confining potential and if the biexciton size was much larger than the exciton size. Here, the extracted ratio $\sim 1.897$ corroborates our assignment of the exciton and biexciton lines. We point out, that in the monolayer $\mathrm{WSe}_{2}$, the free biexciton diameter was expected to be four times larger than the free exciton size ${ }^{28}$, which would translate into a similar ratio.

Resulting from the inherent selection rules of tightly localized excitons, emission from the biexcitonic cascade features a very characteristic polarization behaviour. In fact, if the exciton is subject to a fine structure splitting, induced by structural anisotropies, the biexcitonic emission feature is typically subject to the same splitting, yet with opposite sign. This is a natural consequence of the emission cascade, which is sketched in Fig. 2a. We test the polarization features of our emission pair at low excitation powers. Indeed, for low pump powers, our two primary emission features of interest each split into doublets, which already indicates the presence of a fine structure in our system (Fig. 2b). We carry out polarization-resolved spectroscopy by inserting a linear polarizer and a $\lambda / 2$ wave plate in our beam path and study the intensity of each of these split peaks as a function of the polarizer orientation. Figure $2 \mathrm{c}$ depicts the intensity of each signal, which all feature a strong sinusoidal behaviour, suggesting a close-to perfect linear polarization of both the P1 and the P2 signal. This becomes even clearer as we normalize each peak and plot the corresponding spectra in the contour graph in Fig. 2d. As expected from the emission cascade, the two possible branches of 
a

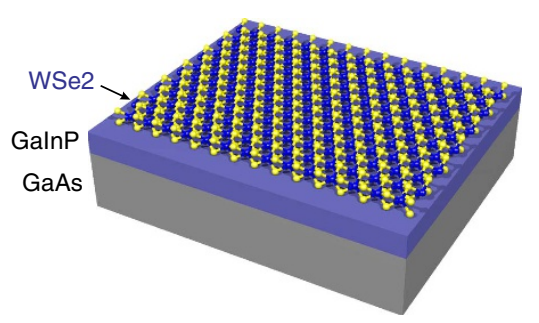

C

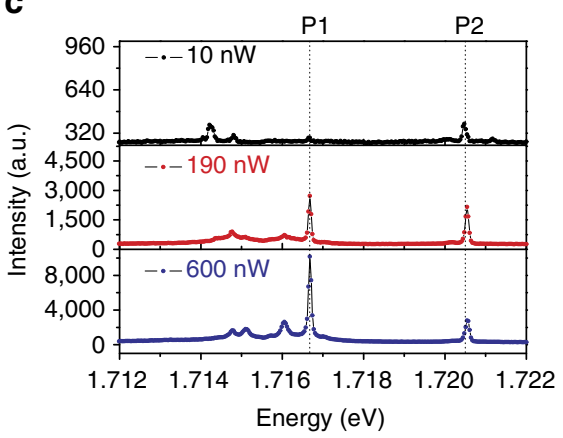

e

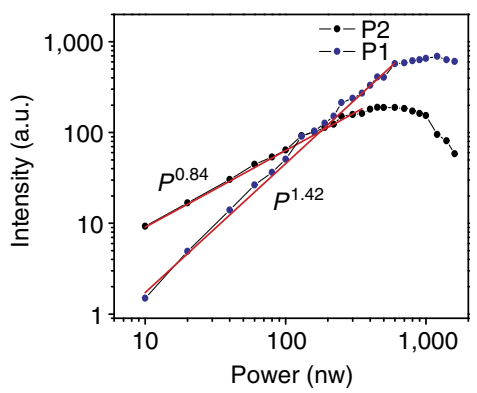

b

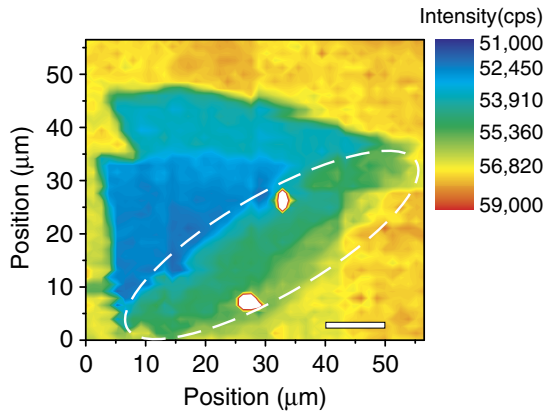

d

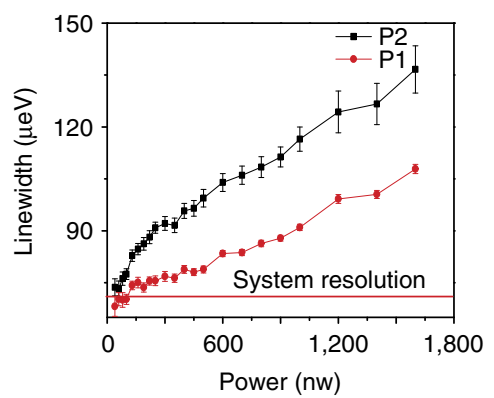

f

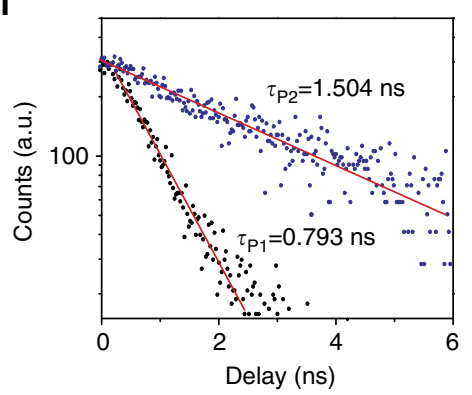

Figure 1 | Narrow spectral lines in monolayer $\mathbf{W S e}_{2}$. (a) Schematic illustration of the device with the deposited WSe $\mathrm{W}_{2}$ monolayer. (b) Contour plot of photoluminescence intensity within the energy range between 1.525 and $1.734 \mathrm{eV}$, over $56 \times 56 \mu \mathrm{m}$. The dashed white line marks the potential monolayer area. (c) Photoluminescence spectrum of the localized emitter in the $\mathrm{WSe}_{2}$ monolayer at $4.5 \mathrm{~K}$, showing different emission behaviours with increasing laser power dominated by peaks at $1.7167 \mathrm{eV}$ (P1) and $1.7206 \mathrm{eV}$ (P2). (d) Extracted linewidth of P1 and P2, plotted as a function of excitation power. The spectrum for low excitation power shows resolution-limited linewidth for both P1 and P2. (e) The integrated counts of the photon emission from P1 and P2 shows super-linear and sub-linear behaviour with increasing laser power in Log-Log plot. The red line is the power law fitting $l \propto P^{x}$, with the extracted $x=0.84$ for $\mathrm{P} 2$ and $x=1.42$ for P1. (f) Time-resolved photoluminescence of P1 and P2 show the single exponential decay with the time constant of $0.793 \pm 0.017 \mathrm{~ns}$ for $\mathrm{P} 1$ and $1.504 \pm 0.028 \mathrm{~ns}$ for P2. Uncertainties are $1 \mathrm{~s} . \mathrm{d}$. values based on a least square fit to the data.

the cascade are separated by the characteristic fine structure splitting both in the $\mathrm{X}$ and the $\mathrm{XX}$ feature with an oscillation period shifted by a phase of $\pi$.

Single-photon correlation spectroscopy. The key hypothesis, namely the presence of an emission cascade from the biexcitonic state to the crystal ground state, can be verified via photon correlation experiments. We prove the capability of our system to emit quantum light by measuring the second-order autocorrelation function of the emission feature P2 under pulse-laser excitation, which we have attributed to the excitonic state. The corresponding autocorrelation histogram from the emission signal is shown in Fig. 3a. The emission is spectrally filtered by a pair of band filters and then coupled into a fibre-based Hanbury-Brown and Twiss setup. Figure $3 \mathrm{~b}$ shows the autocorrelation of the emission P1, which was attributed to the biexcitonic state and filtered out by a monochromator. The results around $\tau=0$ reveal $g^{2}(0)=0.397 \pm 0.04$ for $\mathrm{P} 1(\mathrm{XX})$ and $g^{2}(0)=0.218 \pm 0.06$ for P2 $(\mathrm{X})$, which reach down well below 0.5 and therefore prove single-photon emission from both states. Corresponding measurements under CW non-resonant excitation are shown in Supplementary Fig. 5. Next, we measure the cross-correlation between the emission signals P1 and P2. Figure 3c shows the photon-correlation measurements under the same pulse excitation between two peaks, from which we obtained the secondorder correlation at 0 delay of $g^{2}(0)=1.404 \pm 0.038$ (see Methods for details on the data analysis). The right inset of Fig. $3 c$ shows the cross-correlation between $\mathrm{P} 1$ and $\mathrm{P} 2$ under CW excitation. For $\tau<0$, we observe a characteristic antibunching, which transits to a value significantly above unity at small positive delays. This bunching effect definitely proves the cascaded nature of the singlephoton emission ${ }^{31,32}$ and thus strongly suggests the presence of an emission cascade from a biexciton in our monolayer system.

\section{Discussion}

The microscopic properties of the localized biexciton and its selection rules in monolayers of $\mathrm{WSe}_{2}$ require more work for clarification. The free biexciton states in $\mathrm{WSe}_{2}$ monolayers have 


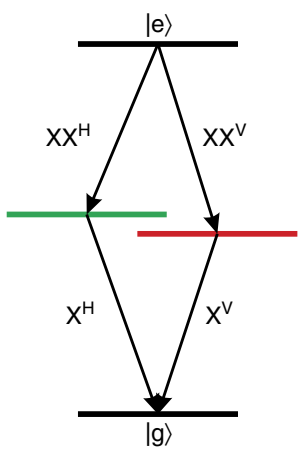

C

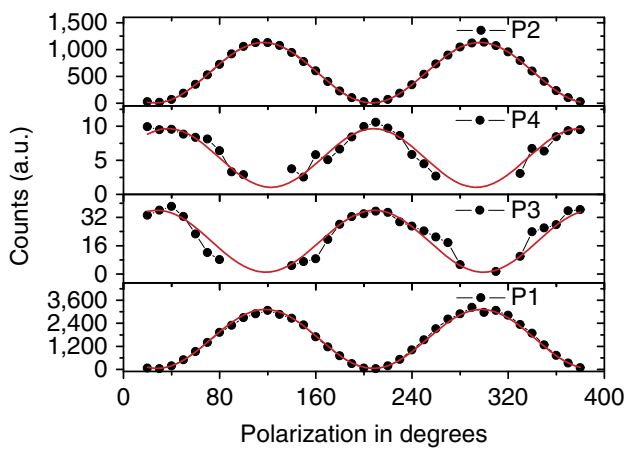

b

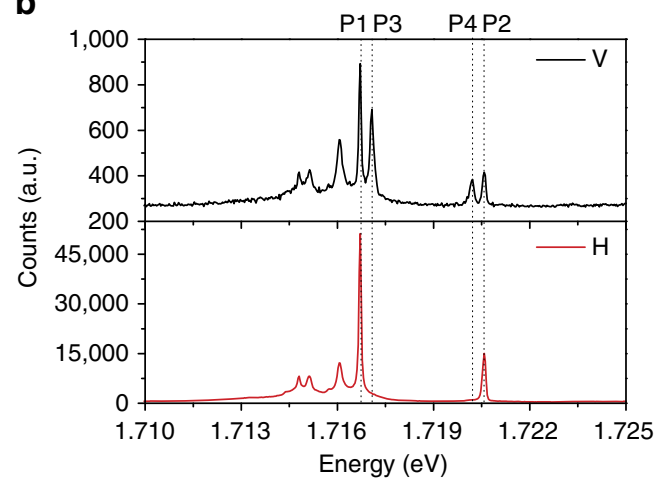

d

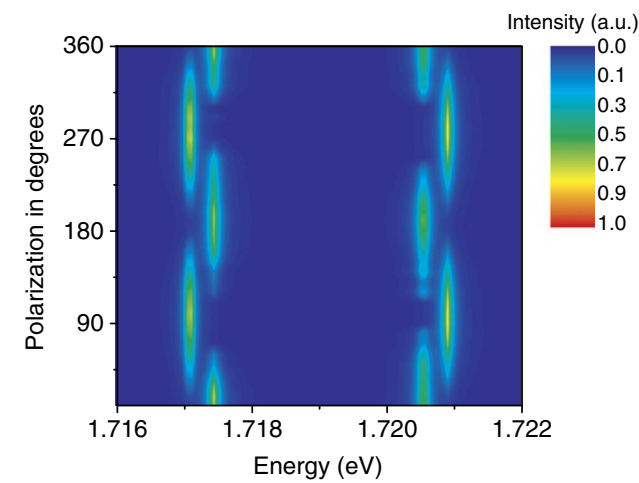

Figure 2 | Polarization-resolved photoluminescence. (a) Schematic representation of biexcitonic emission cascade. The fine-structure splitting is expected for the electron-hole exchange interaction in the presence of in-plane anisotropy. (b) Polarization-resolved spectrum at linear polarization $\mathrm{H}$, V. Two pairs of spectral doublets are observed at 1.7167 (P1)-1.7171 eV (P3) and 1.7202 (P4)-1.7206 eV (P2). Four peaks are indicated by the dashed lines. (c) The integrated counts of the photon emission from P1, P2, P3, P4 as a function of the polarization detection angle. The red lines are the sinusoidal fits, showing two pairs of cross-linear-polarized doublets. (d) Contour representation of the four peaks, after normalizing to the maximum peak intensity, yielding a fine structure splitting $\sim 0.4 \mathrm{meV}$.

been predicted by variational calculations and experimental indications were previously discussed ${ }^{28}$. Similar to the expectations for free biexcitonic states in $\mathrm{WSe}_{2}$, our lifetime measurements indicate that the localized biexcitons are probably larger than the exciton. Furthermore, a previous study of localized excitons in $\mathrm{WSe}_{2}$ indicated the presence of a dark localized exciton and a free dark exciton state several $\mathrm{meV}$ below the bright exciton $^{33,34}$. In our current study, we did not observe any significant contribution of a dark state, which would necessarily lead to a blinking in the defect emission and thus to a long-term bunching in the autocorrelation of the exciton emission. However, it will be interesting to see whether, for example, a temperatureinduced population of the dark exciton will lead to a modification of the observed quantum statistics in our cascaded system.

In conclusion, we have observed clear evidence for the presence of a localized biexcitonic complex in a monolayer of $\mathrm{WSe}_{2}$. The successful observation of the localized biexciton is a consequence of the very clean emission spectra, which in turn are a result of our hybrid semiconductor-monolayer heterostructure. The emission signal features a superlinear increase of its intensity with excitation power, its fine structure splitting is of the same magnitude as the corresponding exciton, yet of opposite linear polarization and, most importantly, the characteristic emission cascade is unambiguously verified in a cross-correlation experiment. Time-resolved spectroscopy on the exciton-biexciton pair suggests that the biexcitonic Bohr radius is approximately four times the size of the exciton's spatial extension. We believe that this demonstration of a multi-particle complex in a monolayer material will give rise to a new plethora of interesting effects, such as the demonstration of valley entanglement. Furthermore, the high optical quality of our emitter on the surface of a sample is a promising step towards the implementation of ultra compact, bright sources of single, indistinguishable ${ }^{35}$ photons and even pairs of polarization and time-bin entangled photons ${ }^{36}$. As the emitting dipole is allocated on a surface rather than embedded in a high index medium, we believe that simple photonic architectures can be developed to obtain broadband quantum light sources with very large photon extraction $^{37}$.

\section{Methods}

Sample design and fabrication. The $\mathrm{WSe}_{2}$ monolayer was mechanically exfoliated from bulk WSe $e_{2}$. We followed the method described in ref. 38. The flake was exfoliated onto a polymer gel film (polydimethylsiloxane) and was then transferred onto a GaAs substrate with $250 \mathrm{~nm}$ GaInP layer on top, where the surface was cleaned with oxygen plasma before the exfoliation.

The GaInP/GaAs substrate was fabricated by gas-source molecular beam epitaxy on epi-ready GaAs (001) substrates. After a thermal oxide-desorption step, a $150 \mathrm{~nm}$-thick GaAs buffer layer was deposited, followed by growth of a $250 \mathrm{~nm}$-thick layer of $\mathrm{Ga}_{0.51} \mathrm{In}_{0.49} \mathrm{P}$ lattice matched to GaAs. The sample was then cooled down under supply of thermally cracked $\mathrm{PH} 3$ gas and removed from the MBE system.

Optical measurements and data analysis. The diagram of the experimental setup is shown in Supplementary Fig. 6 and described in great detail in Supplementary Note 3 . The sample was optically excited non-resonantly using a CW $532 \mathrm{~nm}$ laser and a mode locked, up-converted Ti:Sapphire $(475 \mathrm{~nm})$ with a pulse frequency of $82 \mathrm{MHz}$ and a pulse length of $3 \mathrm{ps}$. A short-pass $(670 \mathrm{~nm})$ filter was inserted into the excitation arm, to filter out the unwanted scattering laser light from the fibre. The collected light was passing through a long-pass $(690 \mathrm{~nm})$ filter. A spectrometer with a CCD (charge-coupled device) cooled by liquid nitrogen was used to measure the photoluminescence. The spectrum was defined by $1,500 \mathrm{~mm}$ 
a

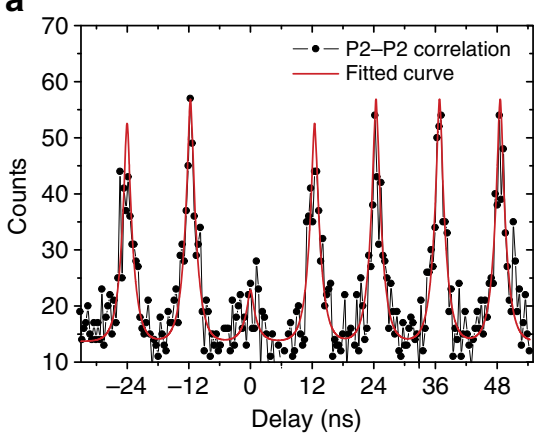

b

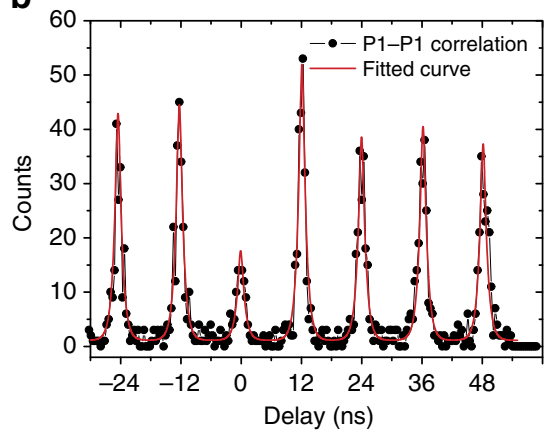

C

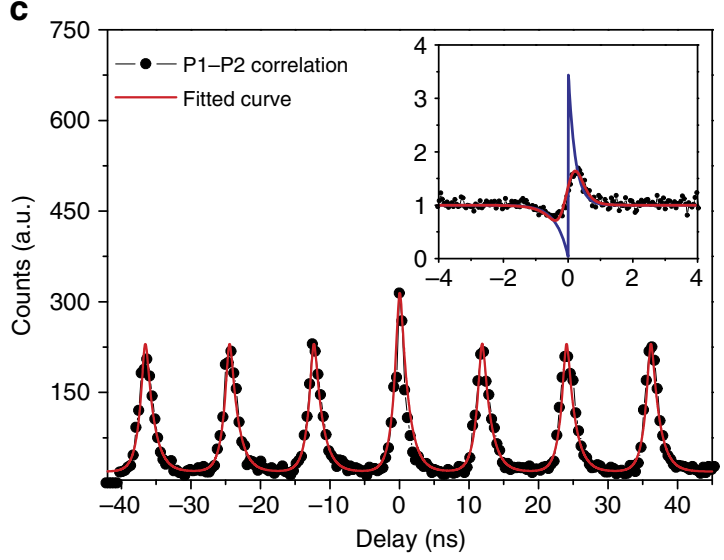

Figure 3 | Photon correlation measurements. (a,b) Second-order autocorrelation of measurement of the P2 and P1 under $13.6 \mathrm{nW}$ pulsed excitation at $475 \mathrm{~nm}$ with the repetition rate of $82 \mathrm{MHz}$ and the pulse length of $3 \mathrm{ps}$. We could extract $g^{2}(0)=0.397 \pm 0.06$ for $\mathrm{P} 1$ and $g^{2}(0)=0.286 \pm 0.04$ for $\mathrm{P} 2$. (c) Cross-correlation measurement between P1 and P2 under pulsed excitation. The obtained $g^{2}(0)=1.404 \pm 0.038$, which obviously shows the bunching effect of the emission. The red lines are fitted with two exponential decay convolved with the response function. The inset is the cross-correlation measurement of P1 and P2 under a $70 \mathrm{nw} C W$ laser excitation at $532 \mathrm{~nm}$. The red line in the inset is a fit with multiexcitonic model convolved with the response function. The blue line is the deconvoluted curve, which shows $g^{2}(0)=0.038 \pm 0.004$ for negative delay and $g^{2}(0)=3.434 \pm 0.129$ for positive delay. All uncertainties correspond to 1 s.d. For more details about fitting, see Methods.

grating with the spectral resolution $\sim 70 \mu \mathrm{eV}$. Photon autocorrelation measurements were performed after collecting the signal into a multimode fibre beam splitter, where the counts were recorded by two single-photon avalanche photodiodes. The correlation function for the pulse experiments was described in the following formula:

$$
g^{2}(t)=y 0+\sum_{n=1}^{n=7} A_{n} \times\left(\exp \left(\frac{t-t_{n}}{\tau_{a}}\right) \Theta(-t)+\exp \left(-\frac{t-t_{n}}{\tau_{b}}\right) \Theta(t)\right)
$$

where $\Theta(t)$ is the step function, $y 0$ is the offset, $A_{n}$ is the peak height of the $n$th peak, $t_{n}$ is the peak position of the $n$th peak. $\tau_{a}=\tau_{b}$ in the exciton (X) autocorrelation and the biexciton (XX) autocorrelation measurement, which are related to characteristic radiation time of $\mathrm{X}$ and $\mathrm{XX}$.

The CW cross correlation data in the inset of Fig. $3 \mathrm{c}$ was fitted with multiexcitonic model similar to that in semiconductor quantum dots (QDs) ${ }^{31}$.

$$
g^{2}(t)=g^{2}(t, \text { neg }) \Theta(-t)+g^{2}(t, \text { pos }) \Theta(t)
$$

Where

$$
g^{2}(t, c)=1-A_{1 c} \times \exp \left(-\left|\frac{t}{t_{1}}\right|\right)-A_{2 c} \times \exp \left(-\left|\frac{t}{t_{2}}\right|\right) .
$$

For $g^{2}(t<0)$, we will see the antibunching, while the bunching for $g^{2}(t>0)$.

To account for the finite timing resolution of our setup, we fitted the pulse correlation data in Fig. 3 with the correlation function convolved with a Gaussian distribution function:

$$
f_{\text {Det }}(t)=\frac{1}{\sigma \sqrt{\pi}} \mathrm{e}^{-\frac{1}{2}\left(\frac{t-t_{0}}{\sigma}\right)^{2}},
$$

where the $2 \sqrt{2 \ln 2} \sigma$ represents the 350 ps time resolution of the avalanche photodiode. So the real fitting function would be:

$$
g_{\text {real }}^{2}(\tau)=\left(g^{2}(t) \times f_{\text {Det }}\right)(\tau)=\int_{-\infty}^{\infty} g^{2}(t) f_{\text {Det }}(\tau-t) \mathrm{d} t
$$

Photon cross-correlation measurements were carried out after passing the signal into a 50:50 beam splitter. One beam was filtered out at P2 by a pair of $1 \mathrm{~nm}$ bandwidth filters centred at $720 \mathrm{~nm}$ and the other beam was leading to the monochromator with the filtered spectrum at P1. The pulsed $g^{2}(0)$ value was calculated from the fitted area in the zero-time delay peak divided by the average of the adjacent six peaks.

Data availability. The data that support the findings of this study are available from the corresponding author upon request.

\section{References}

1. Pan, J. W. et al. Multiphoton entanglement and interferometry. Rev. Mod. Phys. 84, 777-838 (2012).

2. Giovannetti, V. et al. Quantum metrology. Phys. Rev. Lett. 96, 010401 (2006).

3. Georgescu, I. M. et al. Quantum simulation. Rev. Mod. Phys. 86, 153 (2014).

4. Kimble, H. J. The quantum internet. Nature 453, 1023-1030 (2008).

5. Wang, Q. H., Kalantar-Zadeh, K., Kis, A., Coleman, J. N. \& Strano, M. S. Electronics and optoelectronics of two-dimensional transition metal dichalcogenides. Nat. Nanotechnol. 7, 699-712 (2012).

6. Splendiani, A. et al. Emerging photoluminescence in monolayer $\mathrm{MoS}_{2}$. Nano Lett. 10, 1271-1275 (2010).

7. Mak, K. F., Lee, C., Hone, J., Shan, J. \& Heinz, T. F. Atomically thin $\mathrm{MoS}_{2}$ : a new direct-gap semiconductor. Phys. Rev. Lett. 105, 2-5 (2010).

8. Mak, K. F. et al. Tightly bound trions in monolayer $\mathrm{MoS}_{2}$. Nat. Mater. 12, 207-211 (2013).

9. He, K. et al. Tightly bound excitons in monolayer WSe 2 . Phys. Rev. Lett. 113, 26803 (2014)

10. Ross, J. S. et al. Electrical control of neutral and charged excitons in a monolayer semiconductor. Nat. Commun. 4, 1474 (2013).

11. Steinhoff, A. et al. Efficient excitonic photoluminescence in direct and indirect band gap monolayer $\mathrm{MoS}_{2}$. Nano Lett. 15, 6841-6847 (2015). 
12. Chernikov, A. et al. Exciton binding energy and nonhydrogenic Rydberg series in monolayer $\mathrm{WS}_{2}$. Phys. Rev. Lett. 113, 076802 (2014).

13. Ugeda, M. M. et al. Giant bandgap renormalization and excitonic effects in a monolayer transition metal dichalcogenide semiconductor. Nat. Mater. 13, 1091-1095 (2014)

14. Xu, X., Yao, W., Xiao, D. \& Heinz, T. F. Spin and pseudospins in layered transition metal dichalcogenides. Nat. Phys. 10, 343-350 (2014).

15. Wang, G. et al. Valley dynamics probed through charged and neutral exciton emission in monolayer $\mathrm{WSe}_{2}$. Phys. Rev. B 90, 075413 (2014).

16. Xiao, D., Liu, G.-B., Feng, W., Xu, X. \& Yao, W. Coupled spin and valley physics in monolayers of $\mathrm{MoS}_{2}$ and other Group-VI dichalcogenides. Phys. Rev. Lett. 108, 196802 (2012).

17. Jones, A. M. et al. Optical generation of excitonic valley coherence in monolayer $\mathrm{WSe}_{2}$. Nat. Nanotechnol. 8, 634-638 (2013).

18. Zeng, H., Dai, J., Yao, W., Xiao, D. \& Cui, X. Valley polarization in $\mathrm{MoS}_{2}$ monolayers by optical pumping. Nat. Nanotechnol. 7, 490-493 (2012).

19. Mak, K. F., He, K., Shan, J. \& Heinz, T. F. Control of valley polarization in monolayer $\mathrm{MoS}_{2}$ by optical helicity. Nat. Nanotechnol. 7, 494-498 (2012).

20. Cao, T. et al. Valley-selective circular dichroism of monolayer molybdenum disulphide. Nat. Commun. 3, 887 (2012)

21. Dufferwiel, S. et al. Exciton-polaritons in van der Waals heterostructures embedded in tunable microcavities. Nat. Commun. 6, 8579 (2015).

22. Lundt, N. et al. Room-temperature Tamm-plasmon exciton-polaritons with a $\mathrm{WSe}_{2}$ monolayer. Nat. Commun. (in the press).

23. He, Y. M. et al. Single quantum emitters in monolayer semiconductors. Nat. Nanotechnol. 10, 497-502 (2015).

24. Koperski, M. et al. Single photon emitters in exfoliated $\mathrm{WSe}_{2}$ structures. Nat. Nanotechnol. 10, 503-506 (2015).

25. Chakraborty, C. et al. Voltage-controlled quantum light from an atomically thin semiconductor. Nat. Nanotechnol. 10, 507-511 (2015).

26. Srivastava, A. et al. Optically active quantum dots in monolayer $\mathrm{WSe}_{2}$. Nat. Nanotechnol. 10, 491-496 (2015).

27. Tonndorf, P. et al. Single-photon emission from localized excitons in an atomically thin semiconductor. Optica 2, 347-352 (2015).

28. You, Y. et al. Observation of biexcitons in monolayer $\mathrm{WSe}_{2}$. Nat. Phys. 11, 477-481 (2015).

29. Moody, G. et al. Intrinsic homogeneous linewidth and broadening mechanisms of excitons in monolayer transition metal dichalcogenides. Nat. Commun. 6 , 8315 (2015).

30. Bacher, G. et al. Biexciton versus exciton lifetime in a single semiconductor quantum dot. Phys. Rev. Lett. 83, 4417-4420 (1999).

31. Moreau, E. et al. Quantum cascade of photons in semiconductor quantum dots. Phys. Rev. Lett. 87, 183601 (2001).

32. Aspect, A. et al. Time correlations between the two sidebands of the resonance fluorescence triplet. Phys. Rev. Lett. 45, 617-620 (1980).

33. Zhang, X. X. et al. Experimental evidence for dark excitons in monolayer $\mathrm{WSe}_{2}$. Phys. Rev. Lett. 115, 257403 (2015).

34. He, Y. M. et al. Phonon induced line broadening and population of the dark exciton in a deeply trapped localized emitter in monolayer $\mathrm{WSe}_{2}$. Opt. Express 24, 8066-8073 (2016).
35. Vamivakas, A. N. et al. Spin-resolved quantum-dot resonance fluorescence. Nat. Phys. 5, 198-202 (2009).

36. Jayakumar, H. et al. Time-bin entangled photons from a quantum dot. Nat. Commun. 5, 4251 (2014).

37. Lien, D. H. et al. Engineering light outcoupling in 2D materials. Nano Lett. 15, 1356-1361 (2015).

38. Castellanos-Gomez, A. et al. Deterministic transfer of two-dimensional materials by all-dry viscoelastic stamping. 2D Mater. 1, 011002 (2014).

\section{Acknowledgements}

This work has been supported by the State of Bavaria and the European Research Council (Project UnLiMIt-2D). We further thank S. Stoll for assistance in the exfoliation and pre-characterization of the high-quality monolayer material.

\section{Author contributions}

C.S and S.H. initiated the study and guided the work. Y.-M.H. and O.I. carried out spectroscopic investigations. N.L. and O.I. exfoliated, identified and transferred the monolayer. Y.-M.H. and C.S. analysed and interpreted the experimental data, supported by all co-authors. M.D. and K.S. provided the theory. C.S. and Y.-M.H. wrote the manuscript, with input from all co-authors.

\section{Additional information}

Supplementary Information accompanies this paper at http://www.nature.com/ naturecommunications

Competing financial interests: The authors declare no competing financial interests.

Reprints and permission information is available online at http://npg.nature.com/ reprintsandpermissions/

How to cite this article: He, Y.-M. et al. Cascaded emission of single photons from the biexciton in monolayered $\mathrm{WSe}_{2}$. Nat. Commun. 7, 13409 doi: 10.1038/ncomms13409 (2016).

Publisher's note: Springer Nature remains neutral with regard to jurisdictional claims in published maps and institutional affiliations.

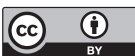

This work is licensed under a Creative Commons Attribution 4.0 International License. The images or other third party material in this article are included in the article's Creative Commons license, unless indicated otherwise in the credit line; if the material is not included under the Creative Commons license, users will need to obtain permission from the license holder to reproduce the material. To view a copy of this license, visit http://creativecommons.org/licenses/by/4.0/

C The Author(s) 2016 